\title{
Image-Based Bed Material Mapping of a Large River
}

\author{
Alexander A. Ermilov ${ }^{1}{ }^{*}$, Sándor Baranya ${ }^{1}$ and Gergely T. Török ${ }^{2}(\mathbb{D}$ \\ 1 Department of Hydraulic and Water Resources Engineering, Budapest University of Technology and \\ Economics, Budapest 1111, Hungary; baranya.sandor@epito.bme.hu \\ 2 Water Management Research Group of the Loránd Eötvös Research Network, Budapest 1111, Hungary; \\ torok.gergely@epito.bme.hu \\ * Correspondence: ermilov.alexander@epito.bme.hu; Tel.: +36-1-463-1687
}

Received: 13 January 2020; Accepted: 20 March 2020; Published: 24 March 2020

\begin{abstract}
The composition or bed material plays a crucial role in the physical hydromorphological processes of fluvial systems. However, conventional bed material sampling methods provide only pointwise information, which can be inadequate when investigating large rivers of inhomogeneous bed material characteristics. In this study, novel, image-based approaches are implemented to gain areal information of the bed surface composition using two different techniques: monocular and stereo computer vision. Using underwater videos, captured in shorter reaches of the Hungarian Danube River, a comparison of the bed material grain size distributions from conventional physical samplings and the ones reconstructed from the images is carried out. Moreover, an attempt is made to quantify bed surface roughness, using the so-called Structure from Motion image analysis method. Practical aspects of the applicability of image-based bed material mapping are discussed and future improvements towards an automatized mapping methodology are outlined.
\end{abstract}

Keywords: bed material mapping; image processing; grain size distribution; structure-from-motion; surface roughness; field measurements; rivers; morphodynamics

\section{Introduction}

There is a permanent interaction mechanism between the water flow and the riverbed, which on the one hand determines the sediment transport capacity of the river yielding to different morphodynamic processes, and on the other hand, the riverbed itself, through the hydraulic resistance, influences the flow features. This back-and-forth effect eventually leads to a complex and continuously changing system of the rivers, possessing great practical importance. For instance, the conditions of fluvial navigation strongly depend on the riverbed morphology in the low water regime, when the ship draught can easily exceed the available flow depth. Bank infiltrated drinking water production depends on, e.g., porosity, stratification, permeability, armoring, colmation, and characteristic sediment grain sizes [1,2]. Moreover, besides the human-related importance, the flow-bed interactions also affect the conditions of aquatic habitats. For example, grain sizes, hiding-factor, inhomogeneity, bedforms, and porosity are all essential for the spawning fish or macroinvertebrates. When analyzing reach scale hydromorphological processes, there is a strong need to gain detailed information on the flow features, the riverbed morphology, the sediment transport, and as a crucial boundary condition: the composition of the riverbed.

Despite its importance, the conventional bed material sampling methods, in a lot of cases, are time-consuming, expensive, and not representative enough, especially when dealing with large rivers of inhomogeneous bed composition. Such problems can easily arise when investigating the transition zones of rivers, i.e., where gravel dominated bed is transformed into sand bed, showing a highly mixed composition, which can vary both in time and space. In these cases, the point-like, local data 
provided by the conventional sampling methods are not adequate. Methods that provide map-like information on the riverbed composition could support a better understanding of the river flow-bed interaction processes.

As digital image-processing and computer software are developing intensively, different methods have already been worked out to estimate grain size distributions and other morphological parameters. One of the increasingly used methods in river morphology measurements is based on the so-called monocular computer vision [3] and is generally divided into two subgroups [4]. One is based on the spectral characteristics or semivariance [5-7], while others use image segmentation [8-10]. As of now, the former seems to be a promising way because these methods require less or no calibration at all [7]. These methods already proved to be usable, not only in the gravel, but sand beds as well. However, they were only used to retrieve point-like information by choosing sampling points along the riverbed. Moreover, they were not yet applied for such river reaches, where the gravel and sand bed create mixed compositions.

Another image-based method belongs to the so-called stereo computer vision [11] group. For river morphological application, the Structure-from-Motion (SfM) method was tested mostly from this category so far. In this method, two or more cameras (or one camera from different directions) record the same area of interest, from which its three-dimensional (3D) model can be created. The method has already been used to reconstruct frozen-core samples [12] to calculate porosity or to create the digital terrain model (DEM) of riverbanks and alluvial fans [13-15] of bedrock and gravel environment, with centimeter-precision. Bed evolution [16] and erosion measurements [14] were also carried out using the DEMs generated with SfM. In laboratory flume, on a gravel bed, statistical roughness analysis was also conducted and compared to other methods, but the obtainable accuracy was lower [17]. So far, most of the roughness studies using SfM were focusing on gravel beds and were mostly carried out in laboratories.

The third group of image-based methods is the application of Artificial Intelligence (AI). Examples can already be seen using Deep Learning Algorithms for landscape classification [18,19]. A great number of drone footages are used to "teach" the algorithm for the basic class-types of the landscape (e.g., gravel, sand, boulder, water, etc.). After a well-established learning-cycle, the algorithm will identify and group new images into the taught categories.

The main goal of this study is to implement image-based bed material analysis methods for field-use performing underwater imaging in a large river. From the above-mentioned three large analysis groups, the monocular and stereo computer vision techniques were tested to see whether the mapping of the riverbed material in reach scale is feasible for future research.

\section{Study Sites}

The field tests were performed along the Hungarian section of the Danube River. This reach of the Danube is a transition zone in terms of bed slope and bed material, where the bed material composition shows strongly varying fractions from silt to gravel even within short distances [20]. For practical reasons, two study sites were chosen, located approximately $100 \mathrm{~km}$ apart. The first study site is located close to the settlement Gönyú between rkm (river kilometer) 1791.2 and 1790.6 (Figure 1). The second one is located at Göd between rkm 1669.4 and 1667.2 (Figure 2). Both study areas can be characterized by an approximately $300 \mathrm{~m}$ channel width, a bed slope of $15 \mathrm{~cm} / \mathrm{km}$, whereas the mean discharges are around $2200 \mathrm{~m}^{3} / \mathrm{s}$ and $1500 \mathrm{~m}^{3} / \mathrm{s}$ (representing the larger branch along a $33 \mathrm{~km}$ long island), respectively. At both sites, 3-3 cross-sections were studied in more detail. 


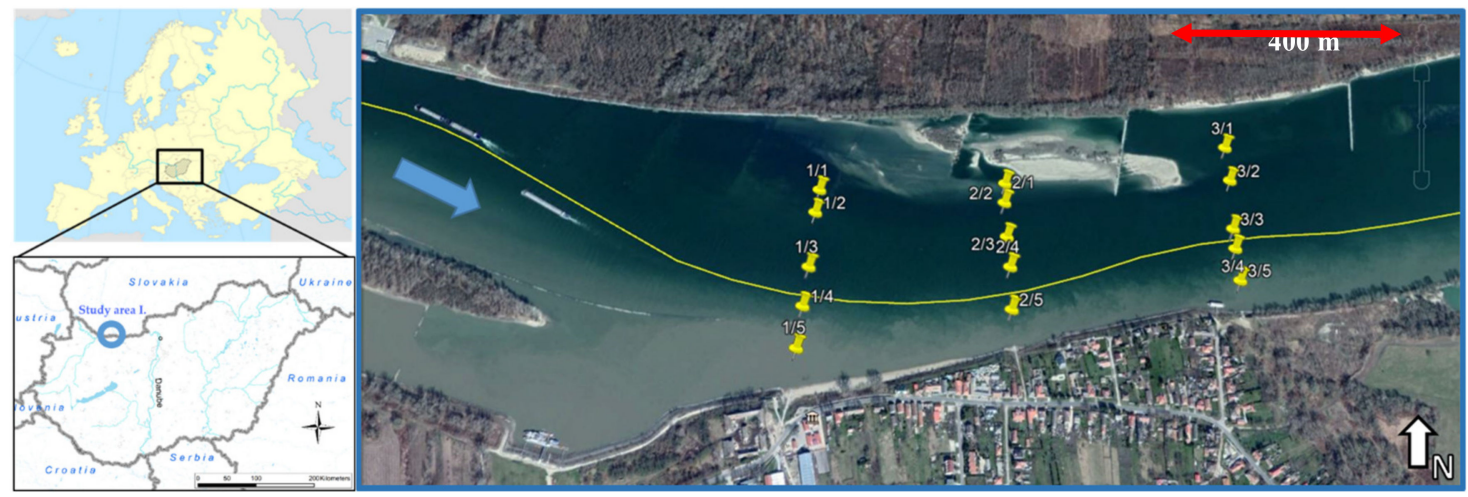

Figure 1. Study area I: Gönyú, Hungary, River Danube (between rkm 1791.2 and rkm 1790.6). Sampling points are marked with yellow pins. Three cross-sections with 5-5 points were selected. Fixed boat measurements were carried out.

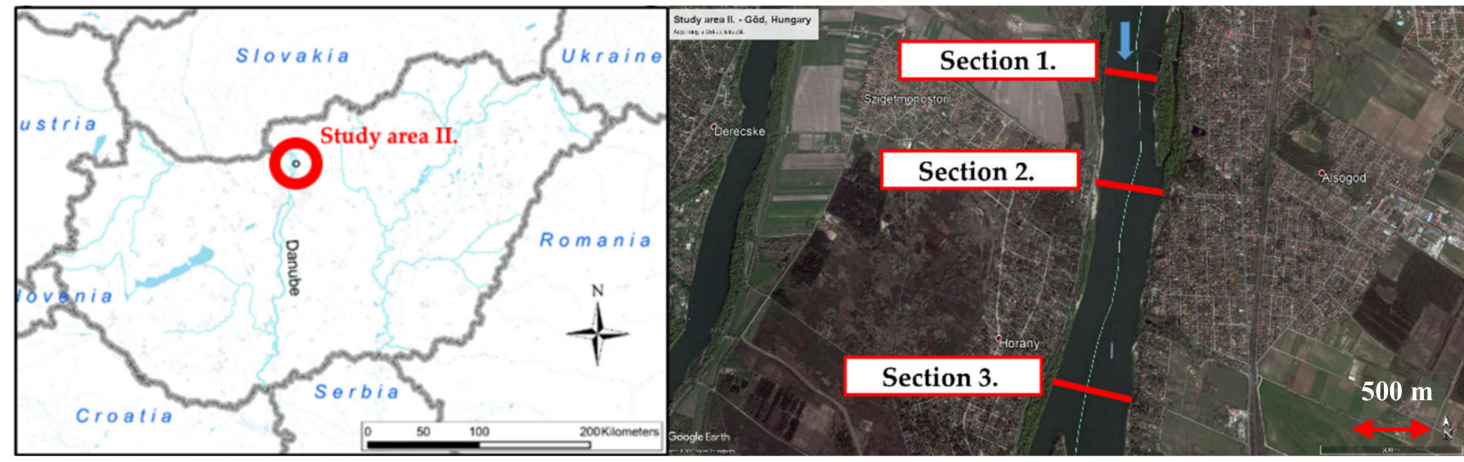

Figure 2. Study area II: Göd, Hungary, River Danube (between rkm 1669.4 and rkm 1667.2). Cross-sectional ADCP (Acoustic Doppler Current Profiler) and video measurements (moving boat measurements) were carried out in the sections marked with red.

\section{Materials and Methods}

The field tests can be separated into two groups. The over-all flowchart of the locations, aims, applied methods, and expected results can be seen in Figure 3. Its elements are further discussed in Sections 3.1 and 3.2 .

\begin{tabular}{|c|c|c|}
\hline & Study area I. & Study area II. \\
\hline Field method & $\begin{array}{c}\text { River bed imaging from fixed } \\
\text { vessel }\end{array}$ & $\begin{array}{c}\text { River bed imaging from moving } \\
\text { vessel }\end{array}$ \\
\hline $\begin{array}{l}\text { Image analysis } \\
\text { method }\end{array}$ & $\begin{array}{c}\text { Monocular approach using } \\
\text { w avelet analysis }\end{array}$ & $\begin{array}{l}\text { Stereo vision approadh using } \\
\text { Structure-from-Motion }\end{array}$ \\
\hline \multirow{5}{*}{ Result } & Grid-by-number GSD & 3D bed surface, surface profile \\
\hline & $\downarrow$ & $\downarrow$ \\
\hline & Shape analysis & Gauss-filtering of the profile \\
\hline & 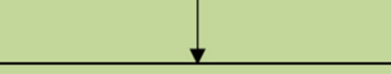 & $\checkmark$ \\
\hline & Volume-by-weight GSD & $\begin{array}{l}\text { 1. Separation of sand and gravel } \\
\text { dominated zones } \\
\text { 2. Bed surface roughness }\end{array}$ \\
\hline
\end{tabular}

Figure 3. Overall flowchart of the measurements and used analysis methods with their expected results. 


\subsection{Applied Monocular Computer Vision Approach for Local Bed Composition Investigation}

First, the applicability of the monocular computer vision approach was investigated at Study site I. Here, videos were captured of the riverbed from which individual images were assessed (Figure 4). A GoPro Hero 4 camera in a waterproof case was attached to a $\sim 50 \mathrm{~kg}$ heavy isokinetic weight, together with a dive flashlight (see the setup in Figure 5). The measurement setup contained a known-sized object fixed right under the camera, aligned with the bottom of the weight so that it touches the riverbed when the setup lands, providing a reference length scale for the image-based analysis (see the screw in Figure 4). The camera was set to $1920 \times 1080$ HD resolution and $48 \mathrm{fps}$.

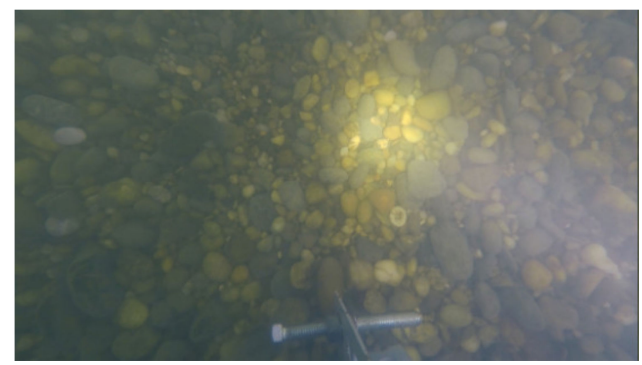

Figure 4. Example of the captured images. Note that a known-sized object was fixed to the instrument setup to provide a reference length scale for further analysis.

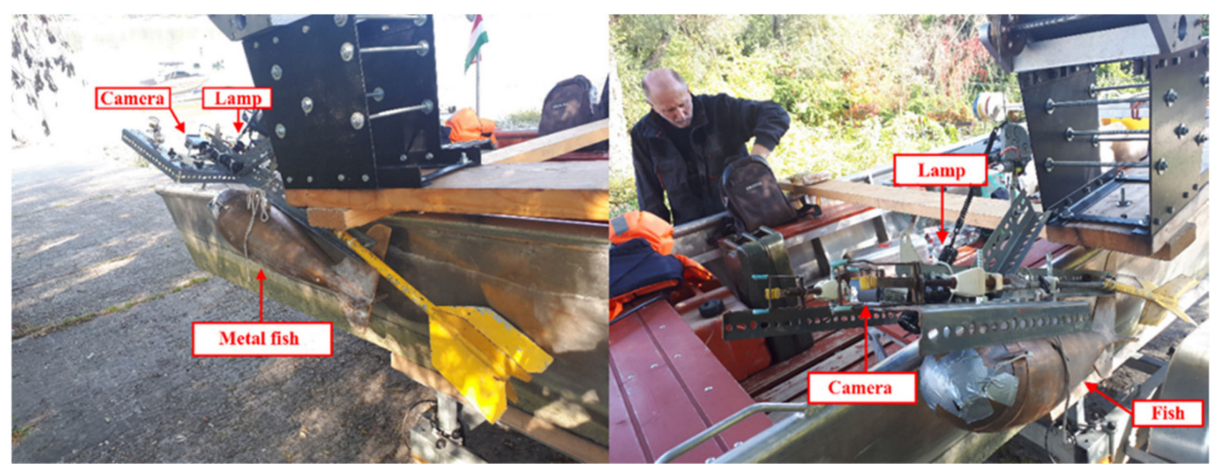

Figure 5. Equipment setup for riverbed imaging: an aluminum frame with an action camera, diving lamp, isokinetic weight.

Besides the imaging, physical bed material samples were also taken, using the conventional bucket sampler in the same verticals, where the videos were recorded. The physical samples were needed for validation purposes of the monocular computer vision approach. The physical bed material samples were brought to the laboratory for drying, and sieving to provide their grain size distributions (GSDs). For the image analysis, the so-called transferable wavelet-method was chosen, using the Matlab code of Buscombe [7], which does not need further calibrations, but the adequate measurement instrumentation. This method converts the input image into a greyscale picture and then analyzes the grey-value (intensity) of each pixel in given rows and columns. For each row and columns, the variation of intensity is then treated as signals. Using continuous Morlet-type wavelet transforms (instead of Fourier transforms), the signals are decomposed, and the power spectra of the transforms are calculated, finally resulting in the grain size distribution in pixels. The goal of this study was to test the method in the field performing underwater imaging in a river reach, where strongly inhomogeneous bed material composition presents. Furthermore, the potential in extending the pointwise information into areal, map-like information of the bed composition was investigated.

To get the GSD in the real length scale, the size of the reference object was used. Eventually, sieving and an image-based GSD could be provided for each sample, enabling the comparison of the physical and indirect methods. However, it is relevant to consider that while sieving gives a volume distribution, the image processing results in an area (or grid-by-number) distribution, so a transformation of one of 
the GSDs was necessary to provide comparable datasets [7,21]. In order to transform the image-based areal information to volume distribution, the characteristic grain shape should be known, i.e., the thickness of the sediment particles. Results from a recent shape analysis were therefore used (Figure 6) based on the categorization method of Zingg, which suggested that the study site can be characterized with disk shape sediments. The average $b / a$ and $c / b$ values of the dataset were 0.76 and 0.62 , respectively (Figure 6, black X). Grains more or less tend to lie with their shortest (c) axes perpendicular to the riverbed, meaning that the intermediate (b) and longest (a) axes are presented in the photos [22]. Hence, we used the intermediate axes retrieved from the image processing, to calculate their other axes by using the average ratio-pairs for disk shape particles (Figure 6, black X). Assuming a constant density of $2.65 \mathrm{~g} / \mathrm{cm}^{3}$, the weight was calculated, providing a by-weight distribution. Performing the previous steps on all the image-based results, the direct comparison of the physical and image-based GSD could be carried out.

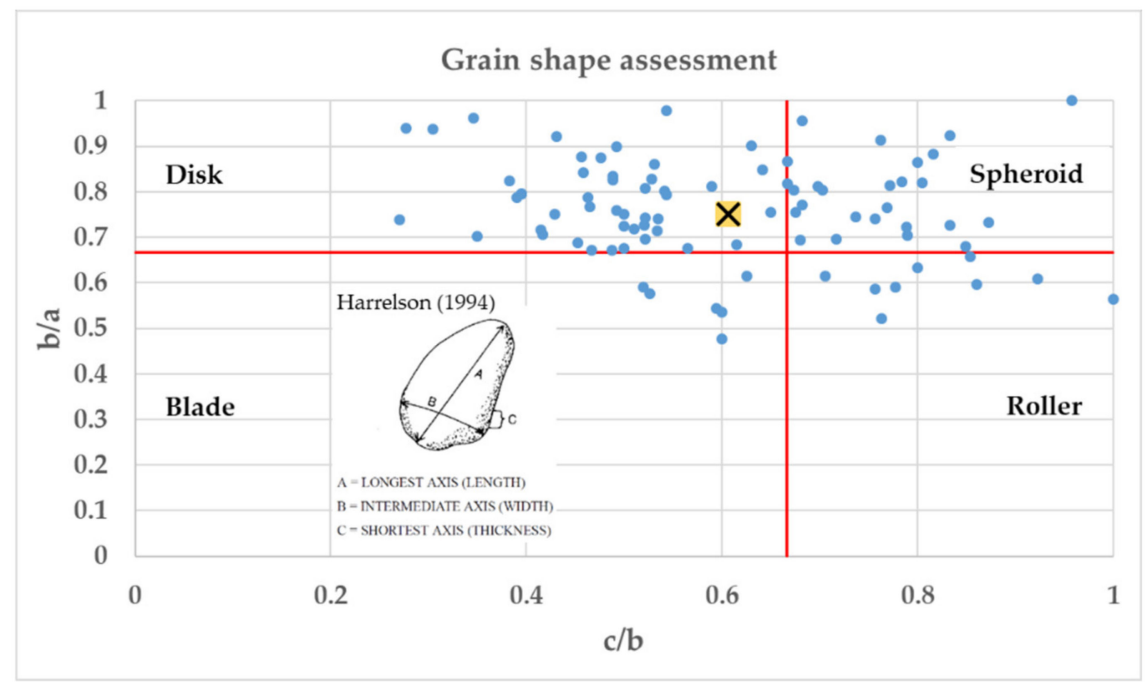

Figure 6. Grain shape assessment results (with blue dots) and the calculated average shape (marked with black X) on a Zingg diagram. The meaning of the axes is also shown. [23].

\subsection{Applied Stereo Computer Vision Techniques for Estimation the Bed Surface Roughness}

In the second part of the study, the stereo computer vision techniques were studied. For this purpose, cross-sectional video-recordings were carried out in study site II by fixing the imaging equipment on a moving vessel. The camera settings were similar to the case of Section 3.1. During the surveys, a real-time kinematic GPS recorded the actual vessel coordinates. In this way, the grain composition information constructed from the images could be located in the absolute coordinate system. The imaging instrumentation used in the first case study (Figure 5) had to be slightly modified due to the fact that the object for scale referencing needed to touch the riverbed. This restraint needed to be avoided, therefore the system was improved using laser lights instead (four laser pointers fixed on the frame), with a known distance between each other. The laser lights could be well-identified on the images, enabling the transformation of the image series from the distorted image coordinate system to a two-dimensional (2D) system.

The image processing method called Structure-from-Motion (SfM) was then applied to the image series. The basic idea of the method is to look for and link the common features, points between the input images. If pictures are taken from different but overlapping angles and directions, it will result in a 3D surface model of the given object. The method is using the Scale Invariant Feature Transform (SIFT) algorithm, known from computer vision, to first retrieve and store the strong features and key points from the images, defining their position, magnitude, orientation, and scale. After this, their neighborhood and other features are examined and paired. As a result, a sparse point cloud is generated [24,25]. The next step includes using one of the Multi-View Stereo (MVS) methods to create 
a dense point cloud [26]. Finally, the points will act as nodes to generate the digital model of the object. Instead of using several cameras for reconstructing the bed surface, we exploited the moving character of the camera, which enabled us to capture images of the same locations from different angles, and sometimes even different distances. For this, the Agisoft Metashape software [27] was used, which is capable of the above-mentioned steps.

Based on the reconstructed surface models, an attempt was made to calculate bed surface roughness. Flow resistance is influenced by skin or grain friction and form drag from developing bedforms. Here, the investigation was carried out for evaluating the former one, by calculating the average roughness height. For this purpose, we followed the theories laid down by mechanical engineering and processing technology, where the surfaces are represented as a composition of different sinusoidal waves. The high frequency waves are defining the roughness, medium frequencies are the waviness, and low frequencies consist of the form of the given surface (Figure 7) [28-31]. The suitable decomposition of the surface profile was performed along transects, using the International Organization for Standardization (ISO) 16610-21 closed profile Gaussian filter. With this approach, the so-called Gaussian mean-line (consisting of the low and medium frequencies) could be produced. Calculating the difference between the raw profile and the Gaussian mean-line gives the roughness profile of the section [28].

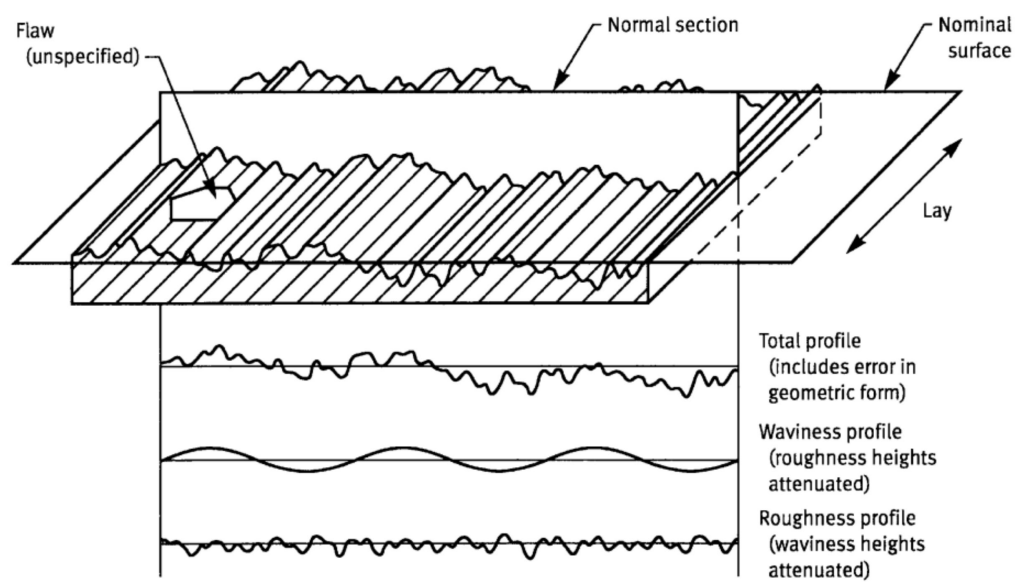

Figure 7. The approach to estimate bed surface roughness from the reconstructed bed surface models: along a normal section, surfaces can be decomposed to high frequency (roughness) and medium frequency (waviness) sinusoidal waves (or even lower frequency ones to get the surface form, not shown in the figure). [30].

For this purpose, one must define the value of the so-called cut-off wavelength $\left(\lambda_{c}\right)$. This value is, in fact, a filter value and is used for the Gaussian-filter function as an input. This parameter distinguishes between roughness (shorter wavelengths than $\lambda_{c}$ ) and waviness (longer wavelengths than $\lambda_{c}$ ). For the suitable determination of this parameter, recommendations are given in the mentioned standard (e.g., it should be at least 2.5 times the average spacing between adjacent peaks of the measured profile on the sampling length). For the calculation, a Matlab code was written, based on the ISO 16610-21 closed profile Gaussian filter [31], and a case-specific cut-off wavelength was chosen, after taking the standard recommendations into account and visualizing the data. After the separation of the roughness profile, the average roughness height $(R a)$ could be calculated with Equation (1) to describe the roughness of the given section:

$$
R a=\frac{1}{L} \times \int_{0}^{L}|Z(x)| d x
$$


where $L[\mathrm{~m}]$ is the sampling length (length of the investigated section) and $Z(x)[\mathrm{m}]$ is the roughness value of the given $x$ point.

\section{Results}

\subsection{Comparative Assessment of Grain Size Distributions}

In the following, a comparison between the GSDs from physical samplings together with the conventional sieving methods and the GSDs constructed from the monocular image-based technique is introduced. As for the latter, two types of composition curves are assessed, i.e., the raw ones resulted directly by the imaging method and the ones after the area-volume transformation, as described above. The median grain sizes $\left(\mathrm{D}_{50}\right)$ and the standard deviations are also assessed.

Depending on the bed composition, the characteristic grain sizes, and shapes, the agreements between the methods show different behavior. There were samples, where the grain size distributions constructed from the images showed a good match with the sieving based GSDs. Such an example can be seen in Figure 8, taken from the sampling point 1/1 (see the sampling location in Figure 1). After transforming the image-based result, the GSD curves showed an even better match on the lower tail of the curves $(<30 \%)$. However, they presented coarser fractions on the higher part. A potential explanation can be that grain-shapes of the coarser fractions differ from the assumed disk-shapes. The standard deviation also showed a better match with the sieving after the transformation, meaning the shapes of the curve are better captured than before the transformation. Thus, in these cases, the fractions above $10 \mathrm{~mm}$ are better estimated without the area-volume transformation.

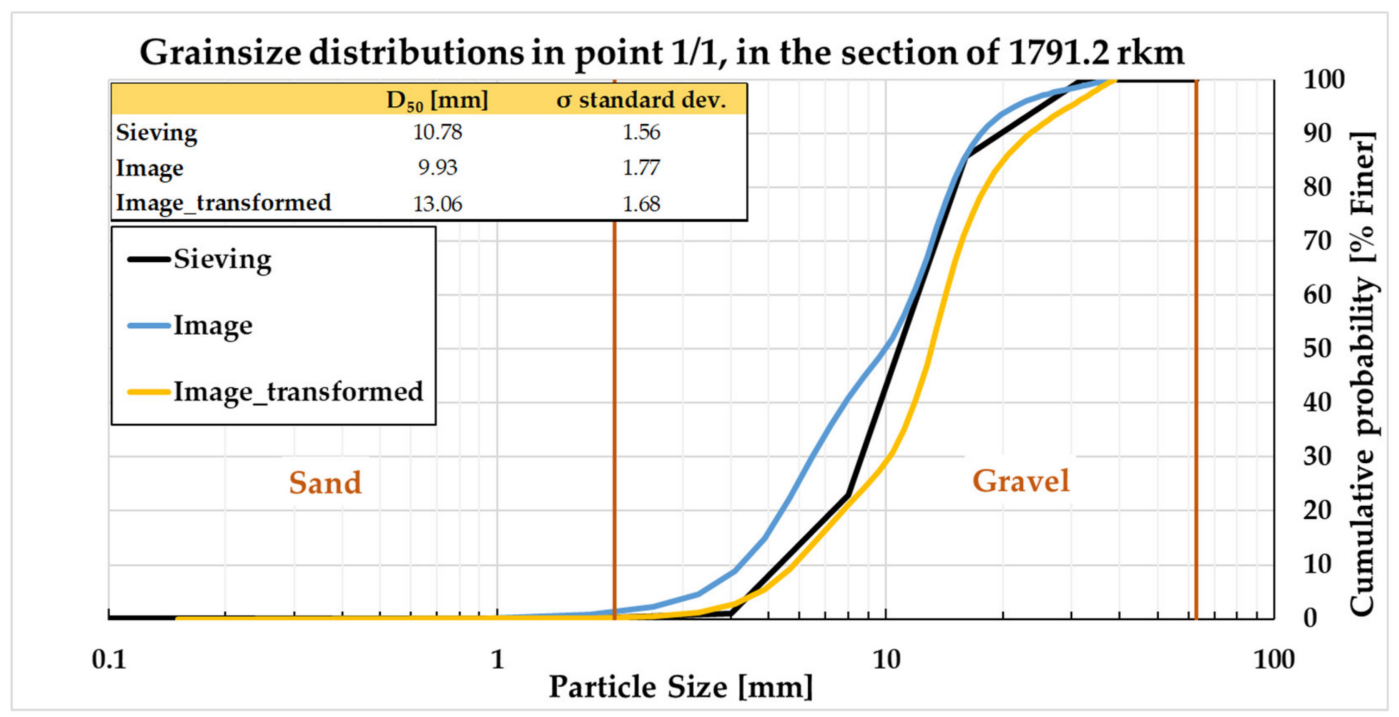

Figure 8. Size distributions of sampling point $1 / 1$. The image-based distribution showed a good match with the sieving results, with the closest matches being on the upper end of the curves. After transforming it, the closest matches turned out to be on the lower tail of the curves, but the shape resemblance became stronger too.

Furthermore, there were several samples where the area-volume transformation improved the agreement, such as in the case of point 2/2 (Figure 9). These samples are generally taken from the coarsest zones of the riverbed, where no sand and fine gravel fractions present. The coarsest fractions are still not captured accurately, but the method works best when no fine fractions can be found on the bed surface. 


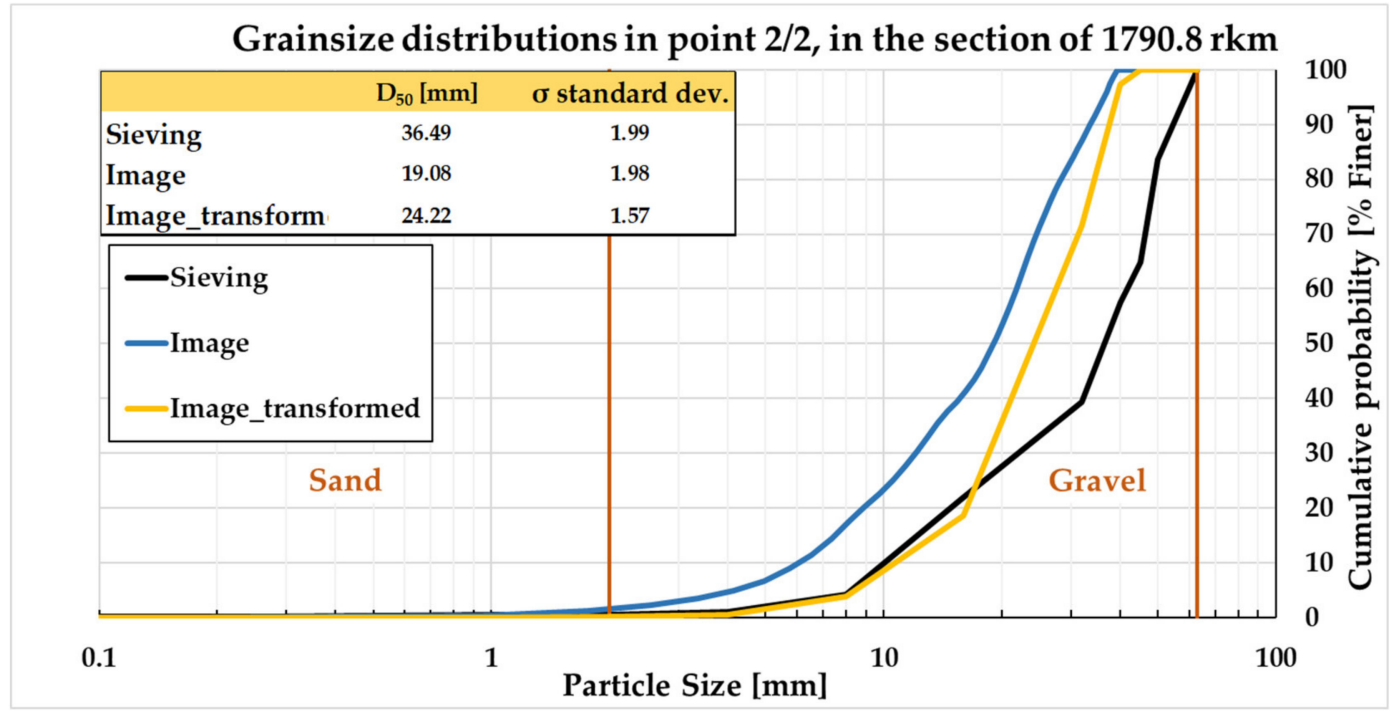

Figure 9. Size distributions of sampling point 2/2. The image-based distribution showed a better match only after transformation.

The most problematic zones are those locations, where fine, sand fractions are present. It is known that in image-based methods, the minimum resolvable grain size depends on the image resolution [4], and the validity of the hereby used digital image analysis method starts from approximately $0.7 \mathrm{~mm}$ (it is 2-3 px normally, [6,32]) due to the applied camera settings and parameters. To see whether the amount of the coarser fractions are well reconstructed by the image-based method, we removed the fractions that were finer than $1 \mathrm{~mm}$ from the sieving based GSD. An example from point $3 / 4$ can be seen in Figure 10. Seeing how the curve moved closer to the image-based curve, we considered it as a proof for the expectations and earlier experience regarding the limitations of the image-based methods (originating from simply resolution limits). The reason for the original image-based GSD showing better match with the sieving (after removing sand), than the transformed result, might be because, in this case, the shortest c-axes (non-visible for the camera) of the grains is not that significant in regards to volume (and mass), so instead of the applied ones, other ratios, or even shape, would be more realistic.

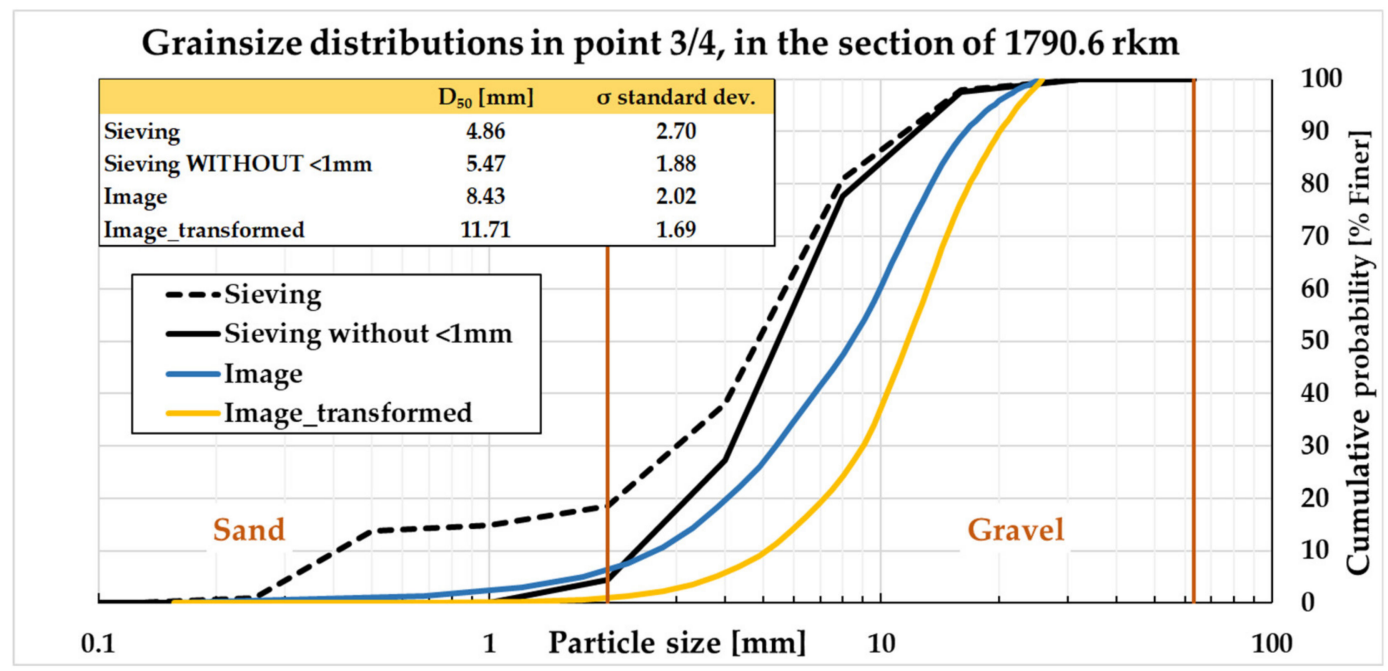

Figure 10. Size distributions of sampling point 3/4. Due to sensing limitations from the applied resolution, both the original and transformed image-based distributions were not matching. By removing the finer than $1 \mathrm{~mm}$ fractions from the sieve, the filtered curved got closer to the image-based curve. 
Involving all the samples, a comparison of the median grain diameter (Figure 11a) as well as the standard deviation values (Figure 11b) were performed between the image-based and sieving based methods. Overall, an adequate agreement was found in the gravel fractions and weak estimation for sand fractions.

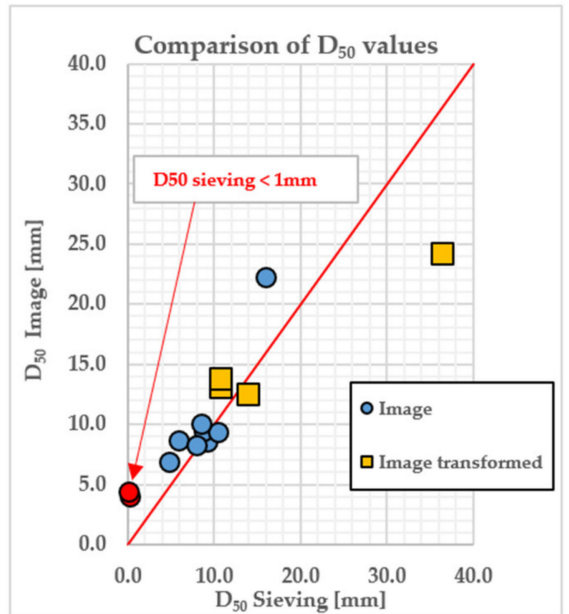

(a)

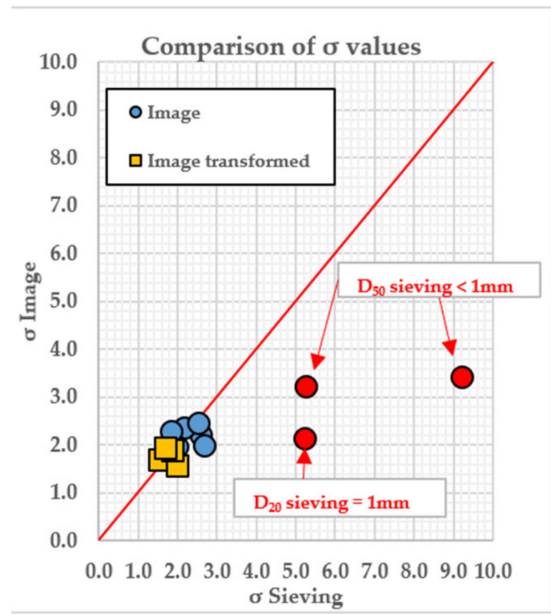

(b)

Figure 11. Comparison of the (a) $\mathrm{D}_{50}$ and (b) standard deviation values from the best fitting (original image distribution or transformed distribution) methods and the sieving results. The sandy points are marked with red.

\subsection{Bed Surface Model and Estimation of Bed Roughness}

As described above, videos captured at the second study site along the river cross-sections were used for performing a stereo computer vision-based, SfM technique. In the following, the results prepared from survey Section 2 (see location in Figure 2) are shown. First, the series of images extracted from the video were merged to provide a large, continuous image of the riverbed for further analysis (Figure 12).

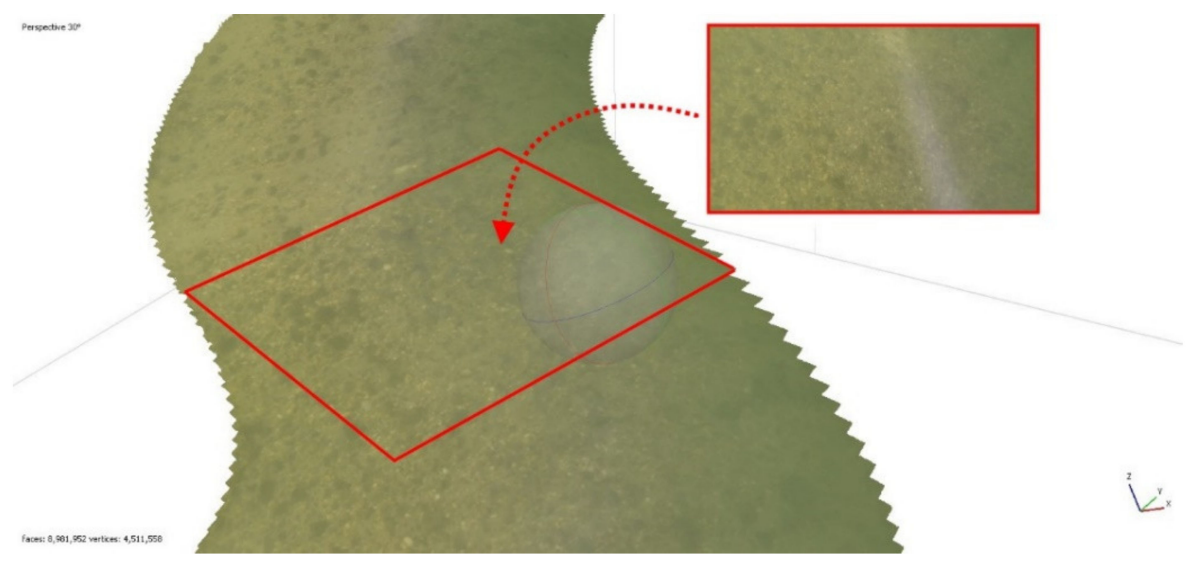

Figure 12. The applied Structure-from-Motion (SfM)-Multiview method analyzed and patched together the frame images of the video. The location and placement of one image can be seen in the figure, along with the modeled surface along the camera path.

For the sake of illustration, only an approximately $21 \mathrm{~m}$ long section (evaluation length) of the whole cross-section is analyzed and showed, but the method, considering the computational demand of the image processing, can certainly be extended for longer profiles. It is worth noting that the computational analysis of this section took approximately $10 \mathrm{~h}$ on a normal PC. After the creation of the bed surface model, a polyline was drawn along the modeled section to extract the total profile 
and calculate the roughness heights. The next step was to use closed profile Gaussian-filter to get the mean-line (low- and medium-frequency elements of the total profile). The difference between the filtered and original profile gives the roughness profile (Figure 13). During the video recording, it was seen that in the middle of this section (from 10 to $12 \mathrm{~m}$ in Figure 13), the riverbed was coarser, gravel dominated than in other parts (e.g., between 2 and $4 \mathrm{~m}$ ), where rather the sand dominated. This phenomenon was also visible after creating the 3D model (Figure 14).
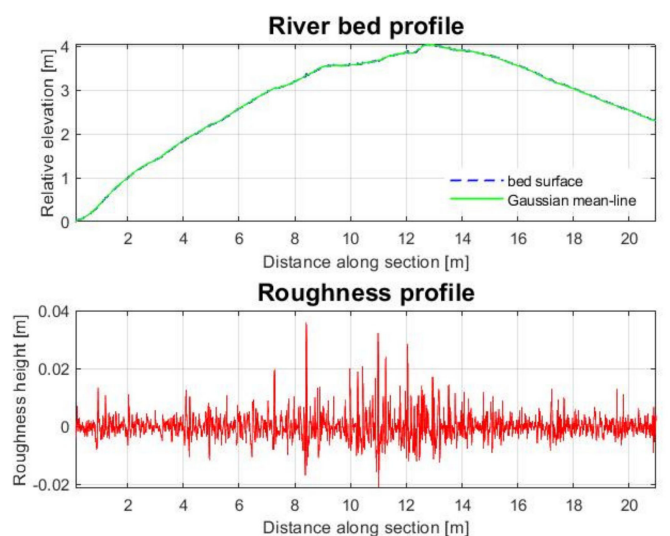

(a)
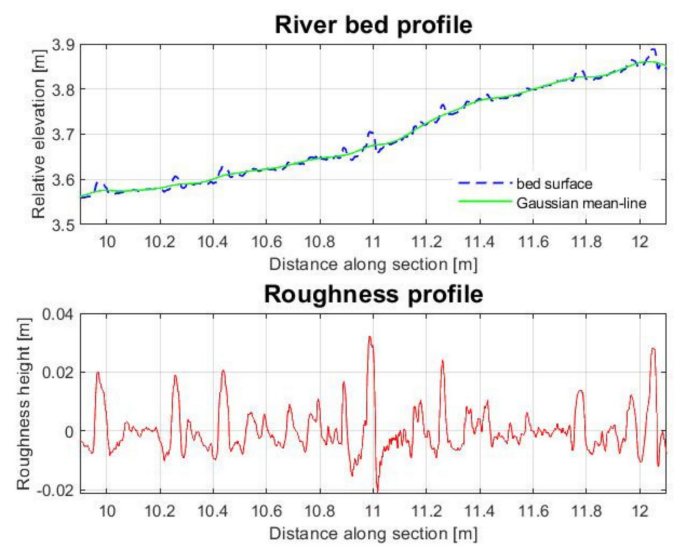

(b)

Figure 13. (a) The bed elevation (top) and the calculated roughness profile (bottom). (b) A coarser part from the analyzed section (middle part in Figure 13a). On (top), the bed surface and the Gaussian mean-line can be seen. From these two, the roughness profile is calculated (bottom).
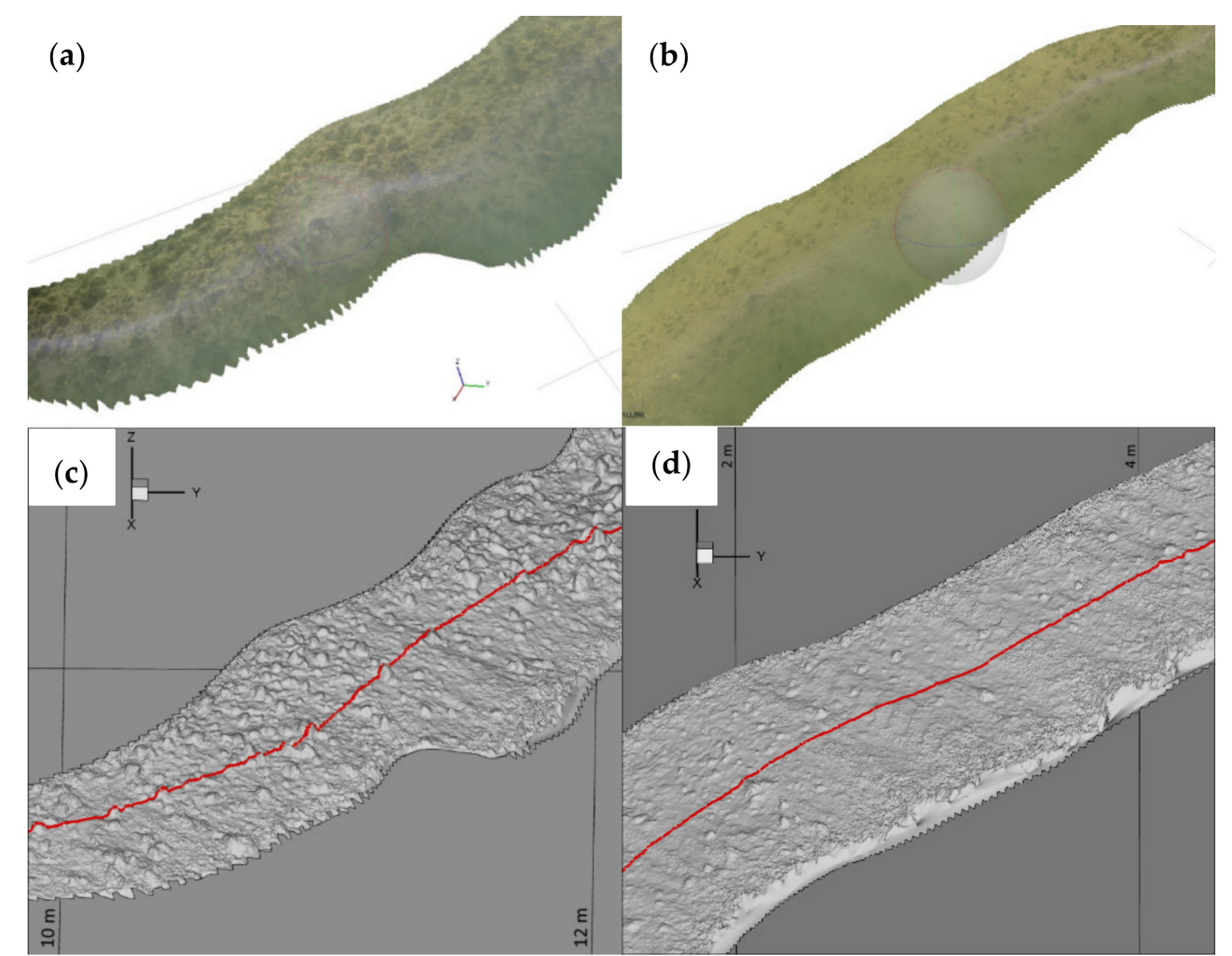

(d)

Figure 14. The coarser part of the section (between 10 and $12 \mathrm{~m}$ along the section) on the left (a,c) and a smoother part on the right (between 2 and $4 \mathrm{~m}$ along the section) $(\mathbf{b}, \mathbf{d})$. The red line is the polyline along which the total profile was retrieved. The pictures on top $(\mathbf{a}, \mathbf{b})$ are the stitched images from the recording, while the pictures on the bottom $(\mathbf{c}, \mathbf{d})$ are the corresponding three-dimensional (3D) models of the given parts. 
The difference in riverbed composition was also visible in the roughness profile (Figure 13). In the coarser part, the total height (vertical distance between a maximum valley and maximum peak) was larger, than in the sand dominated part. For the coarser part $R a_{\text {gravel }}=0.0050 \mathrm{~m}$, for the sand part $R a_{\text {sand }}=0.0017 \mathrm{~m}$, while for the whole $21 \mathrm{~m}$ long section $R a_{\text {total }}=0.0029 \mathrm{~m}$ was calculated, as average roughness height values. Indeed, the riverbed section, where gravel was dominating, the characteristic grain diameter fell in the range of $0.5-1 \mathrm{~cm}$, whereas the typical sand fractions have an order of magnitude lower diameter. This difference in the roughness values could well be captured, underlining that the SfM based fine-scale bed surface model can be used for separating different bed material classes.

\section{Discussion}

In this study, attempts were made to implement image-processing methods for under-water morphological field measurements of large rivers by emphasizing the grain size distribution and roughness of the riverbed as parameters of interest. The field tests were done in the transition zone of the Hungarian section of the Danube River, where the conventional sampling methods are generally inadequate for the representative characterization of bed composition. In contrast with earlier studies applying image-based methods for bed material analysis (e.g., [2-17]), in this study, the investigated river sections show strong inhomogeneity in terms of bed material, ranging from silt to gravel dominated zones. The key question of this research was whether applying a suitable underwater imaging equipment, combining with different image processing methods, could provide areal kind information of the riverbed composition with acceptable quality for further analysis.

As for the developed field imaging equipment, it can be stated that the instrumentation, together with the applied methods, are easy and quick to use. The moving vessel measurements would be the best option for further studies in the future, with the improved instrumentation setup (i.e., lasers), because it can provide adequate input for spatial referencing. It is crucial, however, to note that the introduced underwater imaging is feasible only in low water situations when the water transparency enables clear images. Moreover, there should be no bedload transport during imaging, because the moving particles can bias the resulted grain composition. However, in such circumstances, a suitable image post-processing method could exclude moving particles from the images, as was already introduced by the authors [33].

The applied transferable wavelet-method, as a monocular approach, showed promising results when comparing the constructed GSDs with the ones from sieving analysis. It is, however, important to note that in the case of sand and finer fractions, the method is not able to identify them, resulting in biased GSDs. This insufficiency can be explained with the resolution limitations of image-based methods. To overcome this problem, a combination of the monocular and stereo vision approaches may be necessary, where the latter can account for the separation of fine and coarse particle dominated zones, as discussed later on. The issue of the theoretical differences between sieving and image-based analysis, i.e., volume and areal distribution, was also addressed. A transformation procedure was tested to account for the conversion of areal to volume distributions considering a typical grain shape at the study site. In some cases, the applied estimation gave better results, suggesting that further and more sophisticated involvement of the grain shapes can improve the results.

As for the stereo vision method, a great advantage is the possibility of merging continuously collected images of the riverbed and analyzing grain size distribution for areas with arbitrary sizes, which can eventually yield map-like GSD information. The stereo vision method enables the generation of the 3D model of the riverbed along the camera-path. The herein applied SfM method is reasonably robust. However, it is a key feature to find suitable measurement circumstances in terms of e.g., relative speed of the vessel, camera resolution, framerate, or the distance of the camera from the riverbed. If these parameters are well chosen, this method can indeed provide quantitative information on the bed surface roughness. 
The grain-scale 3D model of the recorded bed surface contains relevant information for a better understanding of flow riverbed interactions but can be of great importance for parameterizing computational flow models. Moreover, as mentioned before, flow resistance is dependent not only on the skin friction, but on the form drag as well. The herein applied filtering method is capable of separating the roughness (skin friction), waviness (forms, form drag), and the channel shape from each other, based on the so-called cut-off wavelengths. Hence, it could be further used in the future for calculating other parameters.

As for future improvements, the exploitation of increasing camera resolution and framerate will lead to more and more accurate assessments with e.g., further decreasing the lower-limit for sensible particle sizes for both the wavelet (GSD) and the SfM (grain-scale 3D model and surface roughness) methods. A logical continuation of the research can be the combination of the wavelet-method with Deep Learning algorithms $[18,19]$ that are capable of recognizing overall textures and patterns. With the combination of the two approaches, it could also contribute to a more accurate separation of sand and gravel patches. Regarding the distribution comparison and grain shape assessment, further studies are planned to carry out more sophisticated transformations of the curves (e.g., not just one average shape and axes-ratios, but different ones for each size class). In case of surface roughness, the average roughness height (root mean square deviation) will be calculated from the presented roughness profiles and attempt will be made to correlate these values with Nikuradse roughness heights and compare the this-way-calculated $D_{50}$ values to ones that are retrieved from sieving or the wavelet method itself. Moreover, besides the presented line roughness (2D), area roughness (3D) [17] will be calculated as well. The final goal would be the automatization of the wavelet-method to retrieve the grain size distributions from any part of the riverbed, mapped by the moving camera(s).

\section{Conclusions}

In this paper, the first results of the assessment of the riverbed composition were introduced based on video imaging of the riverbed. Two different approaches were tested in shorter sections of a large river: monocular and stereo vision methods. The former provided information about the characteristic grain size of the bed surface, whereas the second was used to create fine-scale, 3D bed surface models based on, which, information on the local roughness could be extracted. The results clearly demonstrated the capabilities of both methods, eventually complementing each other. The monocular method showed adequate accuracy for gravel fractions, whereas the grain composition of finer fractions could not be reconstructed well, due to the resolution of the images. On the other hand, the tested stereo vision method could well distinguish between sand and gravel fractions, and so once this separation can be done using the second technique, the first one can be used for more detailed assessment.

The greatest advantage of the introduced image analysis methods is that compared to the conventional, physical bed sampling procedures where local, pointwise information can be extracted from the time-consuming field campaigns, and expensive laboratory analysis, here, after the automatization of the introduced data processing, grain composition information (even along longer streamwise and transversal river transects) can be gained. When the physical characteristics of the bed material strongly vary even in shorter reaches, the quantitative description of these gradients can be crucial.

The results of this study can be well exploited in river engineering problems, when fine scale computational hydro-morphological modelling is performed for shorter river reaches, and map sort of information is needed of the sediment characteristics for model parameterization. Such 3D numerical models are more and more frequently used, e.g., for supporting restoration measures. Furthermore, when, for instance, habitat assessment of rivers is carried out, these image-based methods can also support the description of the varying nature of the benthic zones.

The permanent development of computational capacity, video resolution as well as the new developments in artificial intelligence methods, it is foreseen that these techniques become more and 
more suitable even for large scale analysis of rivers, moreover, the assessment of static images can be further extended to dynamic images, i.e., the quantification of the moving particles and so the investigation of sediment transport on the grain scale can be a realistic goal.

Author Contributions: S.B. conceived and supervised the study. G.T.T. contributed to the preparation of the measurement protocol. A.A.E. put the instrumentation together and carried out the field measurements with the help of technicians. The analyses were done by A.A.E. The results were evaluated by S.B., T.G.T., and A.A.E. together. Conceptualization: S.B.; Data curation: A.A.E.; Formal analysis: A.A.E.; Funding acquisition: S.B.; Investigation: A.A.E.; Methodology: A.A.E.; Project administration: S.B.; Resources: S.B.; Software: A.A.E.; Supervision: S.B. and G.T.T.; Validation, A.A.E.; Visualization, A.A.E.; Writing—original draft, A.A.E.; Writing—review \& editing: S.B. and T.G.T. All authors have read and agreed to the published version of the manuscript.

Funding: The study was partly funded by European Union-European Regional Development Fund via the Interreg Austria-Hungary SEDDON II project. The second author acknowledges the support of the ÚNKP-19-4 New National Excellence Program of the Ministry for Innovation and Technology and the Bolyai János fellowship of the Hungarian Academy of Sciences. This research was partly supported by MTA TKI of the Hungarian Academy of Sciences. Support of grant BME FIKP-VÍZ by EMMI is also kindly acknowledged. The authors acknowledge the funding of the OTKA FK 128429 grant.

Acknowledgments: We would like to thank all the technicians who helped during the field measurements.

Conflicts of Interest: The authors declare no conflict of interest.

\section{References}

1. Rákóczi, L. Identification of River Channel Areas Inclined for Colmation, Based on the Analysis of Bed Material. Vizügyi Közlemények LXXIX; HUNGARICANA: Budapest, Hungary, 1997.

2. Rice, S.; Church, M. Grain size along two gravel-bed rivers: Statistical variation, spatial pattern and sedimentary links. Earth Surf. Process. Landf. 1998, 23, 345-363. [CrossRef]

3. Bassmann, H.; Besslich, P.W. Monocular computer vision. In Proceedings of the Third International Conference on Image Processing and its Applications, Warwick, UK, 18-20 July 1989.

4. Graham, D.J.; Reid, I.; Rice, S.P. Automated sizing of coarse-grained sediments: Image-processing procedures. Math. Geol. 2005, 37, 1-28. [CrossRef]

5. Verdu, J.M.; Batalla, R.J.; Martínez-Casasnovas, J.A. High resolution grain-size characterization of gravel bars using image analysis and geo-statistics. Geomorphology 2005, 72, 73-93. [CrossRef]

6. Buscombe, D.; Masselink, G. Grain size information from the statistical properties of digital images of sediment. Sedimentology 2008, 56, 421-438. [CrossRef]

7. Buscombe, D. Transferable wavelet method for grain-size distribution from images of sediment surfaces and thin sections, and other natural granular patterns. Sedimentology 2013, 60, 1709-1732. [CrossRef]

8. Sime, L.C.; Ferguson, R.I. Information on grain sizes in gravel-bed rivers by automated image analysis. J. Sediment. Res. 2003, 73, 630-636. [CrossRef]

9. Chung, C.-H.; Chang, F.-J. A refined automated grain sizing method for estimating river-bed grain size distribution of digital images. J. Hydrol. 2013, 486, 224-233. [CrossRef]

10. Detert, M.; Weitbrecht, V. User guide to gravelometric image analysis by BASEGRAIN. In Advances in Science and Research; Fukuoka, S., Nakagawa, H., Sumi, T., Zhang, H., Eds.; Taylor \& Francis Group: London, UK, 2013; pp. 1789-1795. ISBN 978-1-138-00062-9.

11. Bebeşelea-Sterp, E.; Brad, R.; Brad, R. A Comparative Study of Stereovision Algorithms. Int. J. Adv. Comput. Sci. Appl. (IJACSA) 2017, 8, 359-375.

12. Seitz, L.; Haas Ch Noack, M.; Silke, W. From picture to porosity of river bed material using Structure-from-Motion with Multi-View Stereo. Geomorphology 2018, 306, 80-89. [CrossRef]

13. Westoby, M.J.; Brasington, J.; Glasser, N.F.; Hambrey, M.J.; Reynolds, J.M. 'Structure-from-Motion' photogrammetry: A low-cost, effective tool for geoscience applications. Geomorphology 2012, 179, 300-314. [CrossRef]

14. James, M.R.; Robson, S. Straightforward reconstruction of 3D surfaces and topography with a camera: Accuracy and geoscience application. J. Geophys. Res. Atmos. 2012, 117, F03017. [CrossRef]

15. Fonstad, M.A.; Dietrich, J.T.; Courville, C.B.; Jensen, J.L.; Carbonneau, P.E. Topographic structure from motion: A new development in photogrammetric measurement. Earth Surf. Process. Landf. 2012, 38, 421-430. [CrossRef] 
16. Bouratsis, P.P.; Diplas, P.; Apsilidis, N. High-resolution 3-D monitoring of evolving sediment beds. Water Resour. Res. 2013, 49, 977-992. [CrossRef]

17. Bertin, S.; Friedrich, H. Measurement of Gravel-Bed Topography: Evaluation Study Applying Statistical Roughness Analysis. J. Hydraul. Eng. 2014, 140, 269-279. [CrossRef]

18. Buscombe, D.; Ritchie, A.C. Landscape Classification with Deep Neural Networks. Geosciences 2018, 8, 244. [CrossRef]

19. Benkő, G.; Baranya, S.; Török, T.G.; Molnár, B. Folyami mederanyag szemösszetételének vizsgálata Mély Tanulás eljárással drónfelvételek alapján (in English: Analysis of composition of river bed material with Deep Learning based on drone video footages). Hidrol. Közlöny 2020, 100, 61-69.

20. Török, T.G.; Józsa, J.; Baranya, S. Validation of a Novel Shear Reynolds Number Based Bed Load Transport Calculation Method for Mixed Sediments against Field Measurements. Water 2019, 11, 2051. [CrossRef]

21. Bunte, K.; Abt, S.R. Sampling Surface and Subsurface Particle-Size Distributions in Wadable Gravel-and Cobble-Bed Streams for Analyses in Sediment Transport, Hydraulics, and Streambed Monitoring; General Technical Report (GTR); U.S. Department of Agriculture, Forest Service, Rocky Mountain Research Station: Fort Collins, CO, USA, 2001. [CrossRef]

22. Warrick, J.A.; Rubin, D.M.; Ruggiero, P.; Harney, J.N.; Draut, A.E.; Buscombe, D. Cobblecam: Grain-size measurements of sand to boulder from digital photographs and autocorrelation analyses. Earth Surf. Process. Landf. 2009, 34, 1811-1821. [CrossRef]

23. Harrelson, C.C.; Rawlins, C.L.; Potyondy, J.P. Stream Channel Reference Sites: An Illustrated Guide to Field Technique; General Technical Report RM-245; U.S. Department of Agriculture, Forest Service, Rocky Mountain Forest and Range Experiment Station: Fort Collins, CO, USA, 1994; p. 61. [CrossRef]

24. Micheletti, N.; Chandler, J.H.; Lane, S.N. Investigating the geomorphological potential of freely available and accessible structure-from-motion photogrammetry using a smartphone. Earth Surf. Process. Landf. 2014. [CrossRef]

25. Micheletti, N.; Chandler, J.H.; Lane, S.N. Structure from Motion (SfM) Photogrammetry, online ed.; In Geomorphological Techniques, British Society for Geomorphology: London, UK, 2015; Chapter 2, Section 2.2, ISSN 2047-0371.

26. Furukawa, Y.; Ponce, J. Accurate dense, and robust multiview stereopsis. IEEE Trans. Pattern Anal. Mach. Intell. 2010, 32, 1362-1376. [CrossRef]

27. Agisoft LLC. Agisoft Metashape User Manual: Professional Edition, Version 1.5. 2019. Available online: https://www.agisoft.com/downloads/user-manuals/ (accessed on 27 February 2019).

28. Muralikrishnan, B.; Raja, J. Computational Surface and Roundness Metrology; British Library Cataloguing in Publication Data; Springer-Verlag: London, UK, 2009; ISBN 9781848002968. [CrossRef]

29. Doi, T.; Marinescu, I.D.; Kurokawa, S. Promising Future Processing Technology. In Advances in CMP Polishing Technologies; Elsevier-William Andrew: Oxford, UK, 2012; Chapter 5; pp. 229-295. [CrossRef]

30. Tavares, S.M.O. Analysis of Surface Roughness and Models of Mechanical Contacts. Master's Thesis, Facoltà di Ingegneria-Università di Pisa, Pisa, Italy, 2005.

31. Vit, J. Surface Metrology Closed Profile Gaussian Filter; MATLAB Central File Exchange: Natick, MA, USA, 2015. Available online: https://www.mathworks.com/matlabcentral/fileexchange/52769-surface-metrology-closedprofile-gaussian-filter (accessed on 9 December 2019).

32. Cheng, Z.; Liu, H. Digital grain-size analysis based on autocorrelation algorithm. Sediment. Geol. 2015, 327, 21-31. [CrossRef]

33. Ermilov, A.A.; Fleit, G.; Zsugyel, M.; Baranya, S.; Török, T.G. Video based bedload transport analysis in gravel bed rivers. In Proceedings of the 21st EGU General Assembly, EGU2019-15071, Vienna, Austria, 7-12 April 2019; Volume 21, p. 1.

(C) 2020 by the authors. Licensee MDPI, Basel, Switzerland. This article is an open access article distributed under the terms and conditions of the Creative Commons Attribution (CC BY) license (http://creativecommons.org/licenses/by/4.0/). 